\title{
Spatial negative priming: Location or response?
}

\author{
W. Trammell Neill ${ }^{1}$ Abigail L. Kleinsmith ${ }^{1}$
}

Published online: 27 July 2016

(C) The Psychonomic Society, Inc. 2016

\begin{abstract}
In tasks requiring a response to the location of a target stimulus (for example, reaching), responses often are slower to a location that was recently occupied by an irrelevant distractor stimulus. In most demonstrations of this "spatial negative priming" (SNP), there is a 1-to-1 correspondence between possible stimulus locations and possible responses. As such, it is ambiguous whether the effect is due to a location-specific processing delay or to inhibition of a response. In the present experiment, subjects were required to press a key corresponding to the ordinal position of a target $\mathrm{O}$ in one of four locations, ignoring a distractor $\mathrm{X}$ appearing in another location. Location markers were widely or narrowly spaced, such that the inner two locations of wide displays corresponded to the outer two locations of narrow displays (hence, requiring different responses). SNP occurred when a target appeared at the location of a recent distractor, regardless of whether the response was associated with the distractor. In contrast, no SNP occurred for a target sharing the same response as a distractor, but in a different location. The results strongly support a location-specific, rather than response-specific, locus of SNP.
\end{abstract}

Keywords Spatial attention $\cdot$ Negative priming $\cdot$ Inhibition of return

Attention to an object typically facilitates responding to a similar or related object that subsequently appears, an effect commonly referred to as priming (Neely, 1977; Posner \&

W. Trammell Neill

wneill@albany.edu

1 Department of Psychology, University at Albany, State University of New York, Albany, NY 12222, USA
Snyder, 1975). However, if an object is initially ignored, responses to a similar or related object often are slower and/or less accurate than responses to an unrelated object-hence, negative priming (Tipper, 1985; Tipper \& Cranston, 1985).

In typical demonstrations of negative priming, a target stimulus is accompanied by a distractor on a prime trial. On a subsequent probe trial, the target stimulus may be similar or related to the prime-trial distractor, or (as a control) be unrelated to it. For example, in the Stroop (1935) color-word task, subjects must name the ink color in which a distractor word is written (e.g., GREEN written in red). Neill (1977) reported that the color-naming response was especially slow if the color corresponded to the distractor word appearing in the previous trial (e.g., BLUE written in green, following GREEN written in red). Similar effects have been shown subsequently in various other tasks, including picture naming, letter naming, letter matching, nonsense-shape matching, and lexical decision (Frings, Schneider \& Fox, 2015; May, Kane \& Hasher, 1995; Milliken, Joordens, Merikle \& Seifert, 1998; Neill, 2007; Neill \& Mathis, 1998; Neill \& Valdes, 1996). There has been considerable debate over what mechanisms cause negative priming; we will defer consideration of these issues to the General Discussion.

In many experiments that demonstrate negative priming, subjects must select one of multiple objects by some criterion, such as location or color, and then identify the object or some attribute of it at that location. Tipper, Brehaut and Driver (1990) noted that we often do the reverse: select by an object's identity and respond to its location - for example, reaching for one's coffee cup on a desk. They demonstrated that negative priming occurs for the location of an irrelevant distractor. In a localization task, subjects pressed a key corresponding to the location of a target symbol (@), while ignoring a distractor symbol (+) appearing in one of three other possible locations. If a probe-trial target appeared in the same location as a prime- 
trial distractor, reaction time (RT) was slower than if the target appeared in a neutral location. This effect is commonly labeled spatial negative priming (SNP), as distinct from identity-specific negative priming (Tipper, Weaver, Cameron, Brehaut \& Bastedo, 1991; Tipper, Lortie \& Baylis, 1992).

As in identity-specific negative priming, there has been debate over the mechanism(s) responsible for SNP (Milliken, Tipper \& Weaver, 1994; Park \& Kanwisher, 1994; Tipper, Weaver \& Milliken, 1995), and by extension, whether SNP and identity-specific negative priming have similar causes (Buckolz, Fitzgeorge \& Knowles, 2012; Christie \& Klein, 2001; Milliken, Tipper, Houghton \& Lupianez, 2000). One area of disagreement has been the locus of processing actually affected by SNP: location or response? Is processing of target information (i.e., perception or identification) hampered at the recently ignored location, or is the response associated to that location inhibited? In most studies of SNP, there has been a 1-to-1 mapping of responses to possible stimulus locations. Consequently, it has been ambiguous whether SNP is location-specific or response-specific.

Neill, Valdes, and Terry $(1992,1995)$ addressed this question in a variation of the localization task, in which four possible stimulus locations were mapped to two responses (left or right hand). A probe target (letter O) might then appear in either the same location as the prime-trial distractor (letter $\mathrm{X}$ ) or in a different location associated to the same response. If SNP were due to response inhibition, RT should be slowed equally for targets at either location. However, RT was significantly slower to a target in the recent distractor location than in the new location. Neill, Valdes and Terry $(1992,1995)$, therefore, concluded that SNP is at least in part location specific. However, as will be elaborated below, a 4:2 mapping of locations to responses does not allow an optimal test of response inhibition. Accordingly, we refrained from any conclusion about whether response inhibition also contributes to SNP.

In contrast, Buckolz, Goldfarb, and Khan (2004) concluded that SNP is response-specific, not location-specific, using similar logic. In their experiment, five locations were mapped to four responses, such that targets at the two leftmost (L1, L2) and two rightmost (L4, L5) locations required unique responses (R1, R2, R3, R4, respectively). If a target appeared at the middle location (L3), subjects had free choice of either R2 or R3. Buckolz et al. found that RT to a probe target at L2 or L4 was slower if a prime-trial distractor appeared at L3 than if the distractor had appeared at L1 or L5. Moreover, RT in the former condition did not differ significantly from that when a prime-trial distractor had appeared in the same location as the probe target (L2 or L4). Insofar as SNP occurred for a target in a different location but requiring one of the responses associated with the distractor location, Buckolz et al. concluded that SNP is responsespecific rather than location-specific.

However, an alternative explanation of the Buckolz et al. (2004) results is consistent with a location-specific interpretation of SNP. Given the nature of the task, it is likely that subjects associated responses with regions rather than with exact points in space. As such, the response-ambiguous location L3 may have been regarded as the same region as L2 or L4 (or both). Consequently, inhibition of processing at L2 or L4 may have generalized to L3. Or from the perspective of memory retrieval theories of negative priming (Neill, Valdes, Terry \& Gorfein, 1992; Neill \& Mathis, 1998), L3 may have been perceived as similar enough to L2 or L4 to cue the retrieval of "ignore this" or "do not respond" to stimuli at that location. In our opinion, the results of Buckolz et al. (2004) do not adequately resolve whether SNP is location-specific or response-specific.

Follow-up experiments by Buckolz and colleagues (Buckolz, Edgar, Kajaste, Lok \& Khan, 2012; Guy, Buckolz \& Khan, 2006) used essentially the same procedure as Neill, Valdes, and Terry (1992; 1995), i.e., a 4:2 mapping of locations to responses. However, they did not find slower RT to a probe target at the prime distractor location than one at a new location associated to the same response. Moreover, RT was slower in the latter condition than in a putative unrelated "control" condition. Consequently, Guy et al. (2006) and Buckolz, Edgar et al. (2012) reiterated the conclusion that SNP is due to response inhibition, with no location-specific contribution.

Unfortunately, we must question whether the 4:2 design used by Guy et al. (2006) and Buckolz, Edgar et al. (2012) — as well as Neill, Valdes, and Terry (1992, 1995) - provides an adequate control condition for response inhibition, on purely logical grounds: In this procedure, the prime target and prime distractor are necessarily assigned to either the same or opposite responses. In practice, two locations to the left of fixation are assigned to one response, and two to the right are assigned to the other response. On a prime trial, the target and distractor either occur on the same side (same response) or opposite sides (different responses). Negative priming cannot be tested in the former condition, because a probe target appearing in the prime distractor location might benefit from response repetition (shared with the prime target). Ergo, conclusions regarding negative priming depend on the subset of trials in which target and distractor are on opposite sides of fixation. What then constitutes a neutral "unrelated" condition? Ideally, a probe target would have to occur at a location not associated with either the prime target or prime distractor. However, there is no such location: If the probe target appears at the "new" location opposite fixation to the prime distractor, then RT may benefit from repeating the same response as the prime 
target. This could give the illusion of "response inhibition" due to the slower RT for the probe target requiring the same response as the prime distractor. ${ }^{1}$

Although the 4:2 design does not test for response inhibition, it remains a valid test of location-specific negative priming. That is, if RT is slower to a probe target at the prime distractor location than to a probe at a new location requiring the same response (and otherwise equated on relation to the prime trial), then there is clearly a location-specific effect. This exact effect was found by Wesslein, Spence, Mast, and Frings (2016) in an investigation of SNP in the tactile domain. In their experiment, subjects responded to one of two possible vibrotactile stimuli applied to the left or right wrist, forearm or upper arm. Critically, one stimulus was designated as a target, the other as a distractor; and, subjects made the same localization response to a target at the wrist or forearm. Wesslein et al. found that RT was slower if a probe target appeared at the location of an ignored distractor than if it appeared at another location mapped to the same response. Consequently, they concluded that SNP in the tactile domain is location-specific, not response-specific.

Ironically, Wesslein et al. (2016) accepted the past conclusions (Buckolz et al., 2004; Guy et al. 2006; Buckolz, Edgar et al., 2012) that SNP in the visual domain is due to response inhibition. As such, there appeared to be a fundamental difference in the cause of SNP between the two modalities (as well as audition). However, as we have argued above, the conclusion that SNP is due to response inhibition may be premature. We report an experiment that provides strong evidence for a location-specific SNP effect, and against a response-specific effect, in the visual modality. Accordingly, the underlying mechanism of SNP in fact may be quite similar between the two modalities.

In the present experiment, we used displays with four location markers, similar to many prior experiments. However, the width of the displays was randomly varied from trial to trial, such that the inner two locations (L2 and L3) of wide displays exactly coincided with the outer two locations (L1 and L4) of narrow displays (Fig. 1). Subjects were required to press a key corresponding to the ordinal position of the target $(\mathrm{O})$, ignoring a distractor $(\mathrm{X})$, regardless of display width.

\footnotetext{
$\overline{{ }^{1} \text { Buckolz et al. }}$ (2004, p. 837) explicitly acknowledged that the experiment by Neill, Valdes, and Terry $(1992,1995)$ was not designed to test for response inhibition. We are surprised, therefore, that follow-up studies (Guy et al., 2006; Buckolz, Edgar et al., 2012) used the same 4:2 design. Although details were not provided, we surmise that their "control" conditions consisted of prime trials in which target and distractor appeared on the same side (i.e., elicited the same response), followed by a probe target on the other side (opposite response). In this case, the probe target is arguably unrelated to the prime target or distractor. However, it cannot be assumed that prime-trial processing is equivalent when target and distractor elicit the same response versus opposite responses. Consequently, these trials do not provide an appropriate control for assessing SNP.
}

Thus, (1) a probe target could appear in the same absolute location as the prime-trial distractor but require a response different from that associated with the distractor; and (2) a probe target could appear in a different absolute location from the prime-trial distractor but require the same associated response. Location-specific SNP predicts slower RT in (1) than in a neutral control condition, whereas response-specific SNP predicts slower RT in (2) than in a neutral control condition. (Of course, both effects are possible a priori.)

\section{Method}

\section{Subjects}

Sixty-four University at Albany undergraduates received experiment participation credit in an introductory psychology course. Each subject was tested individually in a session lasting approximately $40 \mathrm{~m}$.

\section{Subjects and apparatus}

A PC computer running E-Prime E-Studio experiment software, version 1.2, was used to present stimulus displays and to record response latency and accuracy. Subjects executed their responses by pressing the $\mathrm{S}, \mathrm{C}, \mathrm{M}$, and $\mathrm{L}$ keys on the computer keyboard.

Stimulus displays consisted of a central fixation cross, four equally spaced location markers, a target letter $\mathrm{O}$, and (on prime trials) a distractor letter X (see Fig. 1 for example). Wide displays spanned a visual angle of approximately 15 deg; narrow displays spanned a visual angle of approximately 6 deg. Critically, the second and third location markers (L2, L3) of wide displays appeared at the same absolute location as the first and fourth location markers (L1, L4) of narrow displays.

\section{Procedure}

Trials were presented in pairs consisting of a prime trial and a probe trial. A wide or narrow display was randomly selected for each trial, resulting in four types of prime-probe sequence: wide-wide, wide-narrow, narrow-wide, and narrow-narrow. On each trial, a target location was randomly selected from the four possible locations. On each prime trial, one of the remaining locations was randomly selected for a distractor; no distractor was presented on probe trials. ${ }^{2}$ Subjects were instructed to press a key corresponding to the position of the

\footnotetext{
${ }^{2}$ Identity-specific negative priming sometimes depends on a distractor present on the probe trial (Lowe, 1979; Moore, 1994). However, Neill, Terry \& Valdes (1994) found equivalent SNP regardless of whether a distractor appeared in the probe trial.
} 
Example 1

Prime:

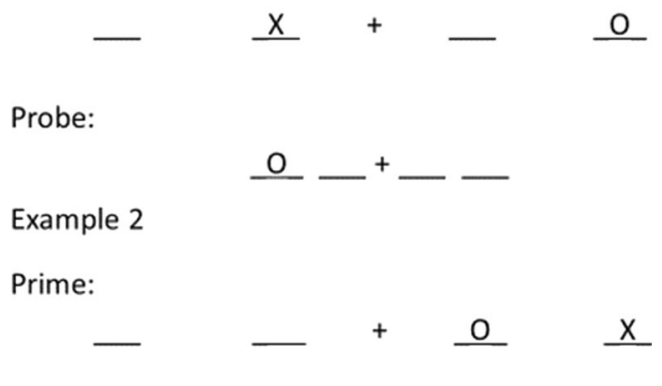

Probe:

$$
\underline{\mathrm{O}}+
$$

Fig. 1 Examples of a wide prime display and narrow probe display. In Example 1, the probe target $(\mathrm{O})$ appears in the same absolute location as the prime distractor $(\mathrm{X})$ but requires a different response than that associated with the distractor location. In Example 2, the probe target $(\mathrm{O})$ appears in a new location that is unrelated to either prime target $(\mathrm{O})$ or prime distractor $(\mathrm{X})$

target $\mathrm{O}$, relative to the four position markers and to ignore the distractor $\mathrm{X}$. The stimulus display remained in view until the subject responded. Incorrect responses were immediately followed by the word ERROR for $400 \mathrm{~ms}$. A blank interval of $600 \mathrm{~ms}$ preceded each trial.

Subjects received five practice trials to become familiar with the response keys. This was followed by 384 primeprobe pairs, subdivided into 6 blocks of 64 pairs each. A 20second rest period separated each block.

\section{Design}

Because selection of display widths, target position, and distractor position (on prime trials) was randomized for each trial, a variety of possible relationships could occur between the prime and probe displays. The probe target could appear in the same location as the prime target (Attended Location); it could appear in the same location as the prime distractor (Ignored Location); it could require the same response as the prime target (Attended Response); or, it could require the same response as that associated with the prime distractor (Ignored Response). If none of these relationships held, then the probe target was Unrelated.

As an example, Fig. 1 (Example 1) depicts a wide prime display with a distractor at L2 and target at L4 and a narrow probe display with a target at L1. (We use L1, L2, L3, and L4 to refer to the ordinal positions, not absolute locations.) Here, the probe target appears in the same absolute location as the prime distractor (Ignored Distractor) but requires a different response than the one associated with the distractor. If the probe target instead appeared at L2, its absolute location would be unrelated to the prime display, but it would require the same response as that associated with the distractor
(Ignored Response). If the probe target appeared at L3, it would not share a location or response with either the prime target or prime distractor (Unrelated). If the probe target appeared at L4, it would be in a neutral location, but require the same response as the prime target (Attended Response).

For same-width sequences (wide-wide and narrownarrow), location repetitions are inherently confounded with response repetitions. By random assignment of targets and distractors, the resultant proportions of different trial types were as follows:

(a) 1/4 Attended Location + Attended Response

(b) 1/4 Ignored Location + Ignored Response

(c) $1 / 2$ Unrelated

For different-width sequences (wide-narrow and narrowwide), the proportions of different trial types were as follows:

(d) 1/24 Ignored Location + Attended Response

(e) 1/24 Attended Location + Ignored Response

(f) 2/24 Ignored Location (different response)

(g) 2/24 Attended Location (different response)

(h) 5/24 Ignored Response (different location)

(i) $5 / 24$ Attended Response (different location)

(j) 8/24 Unrelated (including 2/24 "location controls")

It may be noted that there are actually two types of Unrelated trials. With reference to Fig. 1 (Example 1), a probe target at L3 appears at a location that was not possible in the prime trial. In other sequences, an unrelated probe target could appear at a location that was possible in the prime trial as well. Figure 1 (Example 2) illustrates a sequence in which the probe target at L1 appears at L2 of the prime display and is unrelated to either prime target or prime distractor. Because location repetitions by definition imply a location possible in both prime and probe, the latter unrelated probes provide a more precise control condition for location-specific effects of repetition. We will refer to these trials as "location controls." It may be noted that response repetitions can occur in locations either possible or not possible in the prime (compare L4 in Fig. 1, Example 1, and L3 in Example 2). Consequently, all Unrelated trials were used as a control for response-specific effects.

\section{Results}

The results for Attended Location and Attended Response trials are not directly pertinent to the issues concerning SNP. For ease of exposition, we will only briefly summarize those results. (Details may be obtained from the authors.) Attended Location yielded facilitation relative to Unrelated in the wide-wide and narrow-narrow 
sequences, i.e., when the repeated locations required the same responses. Attended Location yielded significantly slower responses than Unrelated in the wide-narrow and narrow-wide sequences, in which repeated locations required different responses. These results are unsurprising, on the supposition that repetition of a target location would tend to elicit the same response; that response would interfere with executing a different response. Similarly, Attended Response yielded facilitation only in the wide-wide and narrow-narrow conditions, in which the target location was repeated. There was no benefit (or cost) to repeating the same response to different target locations, i.e., in the wide-narrow and narrow-wide sequences.

Table 1 displays the mean RT for correct responses, and percentage of error responses, for Ignored Location/Response and Unrelated trials in wide-wide and narrow-narrow sequences. In these sequences, Ignored Location was redundant with Ignored Response. As shown, pairwise $t$ tests yielded significant negative priming in both RT and errors for both types of sequence.

Table 2 displays the mean RT for correct responses, and percentage of error responses, for Ignored Location and Unrelated (location control) trials in wide-narrow and narrow-wide sequences. In these sequences, Ignored Location effects are "pure," i.e., not conflated with Ignored Response (or Attended Response). As shown, pairwise $t$ tests again yielded significant negative priming in both RT and errors for both types of sequence.

Table 3 displays the mean RT for correct responses, and percentage of error responses, for Ignored Response and Unrelated trials in wide-narrow and narrow-wide sequences. In these sequences, Ignored Response Effects are "pure," i.e., not conflated with Ignored Location (or Attended Location). In contrast to the data shown in Tables 1 and 2, pairwise $t$ tests yielded no significant negative priming. In fact, error percentages revealed significant positive priming for responses associated with the ignored distractor.

Table 1 Mean RT and \% errors in wide/wide and narrow/narrow sequences: location repetition confounded with response repetition

\begin{tabular}{|c|c|c|c|c|}
\hline \multirow[b]{3}{*}{ Relation } & \multicolumn{4}{|c|}{ Prime/probe display sequence } \\
\hline & \multicolumn{2}{|c|}{ Wide/wide } & \multicolumn{2}{|c|}{ Narrow/narrow } \\
\hline & RT & $\% \mathrm{E}$ & RT & $\% \mathrm{E}$ \\
\hline Ignored & 581 & 4.1 & 557 & 3.8 \\
\hline Unrelated & $\underline{557}$ & 2.4 & $\underline{547}$ & $\underline{2.4}$ \\
\hline Negative priming & $24 * *$ & $1.7^{*}$ & $9 *$ & $1.3^{*}$ \\
\hline
\end{tabular}

$* p<0.05 ; * * p<0.01$ (two-tailed)
Table 2 Mean RT and \% errors in wide/narrow and narrow/wide sequences: location repetitions without response repetitions

\begin{tabular}{|c|c|c|c|c|}
\hline \multirow[b]{3}{*}{ Relation } & \multicolumn{4}{|c|}{ Prime/probe display sequence } \\
\hline & \multicolumn{2}{|c|}{ Wide/narrow } & \multicolumn{2}{|c|}{ Narrow/wide } \\
\hline & RT & $\% \mathrm{E}$ & RT & $\% \mathrm{E}$ \\
\hline Ignored & 598 & 6.2 & 571 & 6.1 \\
\hline Unrelated & $\underline{565}$ & $\underline{3.7}$ & $\underline{556}$ & $\underline{3.2}$ \\
\hline Negative priming & $33^{* *}$ & $2.4^{*}$ & $15^{* *}$ & $2.9 * *$ \\
\hline
\end{tabular}

$* p<0.05 ; * p<0.01$ (two-tailed)

\section{Location analyses}

We submitted the RT and error data shown in Tables 1 and 2 to $2 \times 2 \times 2$ repeated-measures analyses of variance (ANOVA) with variables of prime width (wide vs. narrow), probe width (wide vs. narrow) and relation (Ignored Location vs. Unrelated). The data from wide-narrow and narrow-wide sequences (Table 2) reflect "pure" location repetition effects without response repetitions; the inclusion of the data from wide-wide and narrow-narrow sequences (Table 1) allows an assessment of whether location-repetition effects are moderated at all by response repetitions, as manifested in prime width $\mathrm{x}$ probe width interactions.

RT was faster following a narrow prime $(\mathrm{M}=558 \mathrm{~ms})$ than following a wide prime $(575 \mathrm{~ms}), \mathrm{F}(1,63)=33.34, p<0.0005$, MSe $=1,174$. This effect was moderated by an interaction with probe width, $F(1,63)=14.36, p<0.0005$, MSe $=$ 1,194 , reflecting faster RT overall when prime and probe width were the same $(561 \mathrm{~ms})$ than when different $(572 \mathrm{~ms})$. Of more relevance to the present hypotheses, Ignored Location $(577 \mathrm{~ms})$ was slower overall than Unrelated (556 ms), $F(1,63)=37.18, p<0.0005$. MSe $=1,437$. This negative priming effect interacted with prime width, $F(1,63)=$ 6.53, $p=0.013$, MSe $=1,283$. As shown in Tables 1 and 2, SNP was greater following wide primes $(29 \mathrm{~ms}$, overall) than following narrow primes $(12 \mathrm{~ms})$. (This might simply reflect a floor effect, i.e., the faster RT following narrow primes attenuating condition differences.) However, the prime width $\mathrm{x}$ probe width $\mathrm{x}$ relation interaction did not approach significance, $F(1,63)=1.54, p=0.219$. In other words, the conflation of Ignored Location with Ignored Response in wide-wide and narrow-narrow sequences (Table 1) did not increase SNP relative to Ignored Location alone in wide-narrow and narrow-wide sequences (Table 2). Indeed, the SNP effects are quantitatively larger for the "pure" location effects shown in Table 2.

A similar analysis of error percentages again yielded an interaction of prime width with probe width, $F(1,63)=$ $13.88, p<0.0005, \mathrm{MSe}=0.002$, reflecting fewer errors overall when prime and probe displays were same width (3.2\%) 
Table 3 Mean RT and \% errors in wide/narrow and narrow/wide sequences: response repetitions without location repetitions

\begin{tabular}{llllll}
\hline & \multicolumn{2}{l}{ Prime/probe display sequence } \\
\cline { 2 - 3 } & \multicolumn{2}{l}{ Wide/narrow } & & \multicolumn{2}{l}{ Narrow/wide } \\
\cline { 2 - 3 } \cline { 5 - 6 } Relation & RT & $\% \mathrm{E}$ & & $\mathrm{RT}$ & $\% \mathrm{E}$ \\
\hline Ignored & 578 & 3.7 & & 566 & 2.4 \\
Unrelated & $\frac{576}{2}$ & $\frac{5.9}{-2.2} * *$ & & $\frac{563}{3}$ & $\frac{5.6}{-3.3 * *}$ \\
Negative priming & 2 & & & & \\
\hline
\end{tabular}

$* p<0.05 ; * * p<0.01$ (two-tailed)

than different width (4.8\%). More errors occurred overall for Ignored Location $(5.0 \%)$ than Unrelated $(2.9 \%), F(1,63)=$ $23.55, p<0.0005, \mathrm{MSe}=0.002$. This negative priming effect did not interact with prime or probe width. Notably, the prime width $\mathrm{x}$ probe width $\mathrm{x}$ relation did not approach significance, $F(1,63)=1.70, p=0.198$, again suggesting that SNP did not depend on the conflation of Ignored Location with Ignored Response in the wide/wide and narrow/narrow conditions. As in the RT data, the SNP effects are actually larger quantitatively for the "pure" location effects shown in Table 2.

\section{Response analyses}

We submitted the RT and error data shown in Tables 1 and 3 to $2 \times 2 \times 2$ repeated-measures analyses of variance (ANOVA) with variables of prime width (wide vs. narrow), probe width (wide vs. narrow), and relation (Ignored Response vs. Unrelated). The data from wide-narrow and narrow-wide sequences (Table 3) reflect "pure" response repetition effects without location repetitions; the inclusion of the data from wide-wide and narrow-narrow sequences (Table 1) allows an assessment of whether response-repetition effects are moderated at all by location repetitions, as manifested in prime width $\mathrm{x}$ probe width interactions.

As in the location analyses, RT was slower overall following wide primes $(573 \mathrm{~ms})$ than narrow primes $(558 \mathrm{~ms})$, $F(1,63)=45.33, p<0.0005, \mathrm{MSe}=613$; this effect interacted similarly with probe width, $F(1,63)=14.76, p<0.0005$, MSe $=917$. Again, RT was faster when prime width and probe width were same $(561 \mathrm{~ms})$ than when different $(571 \mathrm{~ms})$. RT was slower overall for Ignored Response $(571 \mathrm{~ms})$ than Unrelated $(561 \mathrm{~ms}), F(1.63)=23.92, p<0.0005$, MSe $=$ 489. Unlike the location analyses, the prime-width $\mathrm{x}$ probewidth $\mathrm{x}$ relation interaction was significant, $\mathrm{F}(1,63)=7.21, p$ $=0.009, \mathrm{MS}=941$, reflecting a negative priming effect only when response repetition was confounded with location repetition (wide-wide and narrow-narrow sequences; Table 1).

ANOVA for error percentages also yielded the prime width $\mathrm{x}$ probe width interaction, $F(1,63)=12.65, p=0.001$, $\mathrm{MSe}=$ 0.002 , reflecting fewer errors when prime width and probe width were the same $(3.2 \%)$ than when different $(4.4 \%)$. The overall difference between Ignored Response $(3.5 \%)$ and Unrelated (4.1\%) was significant, $F(1,63)=4.37, p=$ $0.041, \mathrm{MSe}=0.001$, but in the direction of positive priming. Moreover, the prime width $\mathrm{x}$ probe-width $\mathrm{x}$ relation interaction was again significant, $\mathrm{F}(1,63)=36.55, p<0.0005$, $\mathrm{MSe}=$ 0.002 , reflecting a negative priming effect when response repetition was confounded with location repetition (wide-wide and narrow-narrow; Table 1), but positive priming for "pure" response repetition effects (wide-narrow and narrow-wide; Table 3).

\section{Discussion}

In most experiments investigating spatial negative priming (SNP) in localization tasks, there has been a 1-to-1 mapping of possible stimulus locations to the corresponding responses (Neill, Terry \& Valdes, 1994; Tipper, et al., 1990, 1991, 1992). As such, it is impossible to distinguish between impairment of processing at the recent distractor location versus inhibition of the response associated with that location. Some past experiments have tried to resolve this issue (Buckolz et al., 2004; Buckolz, Edgar et al., 2012; Guy et al., 2006; Neill, Valdes \& Terry, 1992), with arguably inconclusive results. The present experiment strongly supports the conclusion that SNP is location-specific, not response-specific.

By varying display widths, we were able to test for SNP for a probe target at the location of an ignored distractor, but associated with a different response, and SNP for a probe target requiring the response associated with the ignored distractor but at a different location. Only the former condition yielded SNP, and this effect was statistically indistinguishable from conditions in which location and response were confounded. In contrast, there appeared to be no cost to making a response that was associated with the ignored distractor, if its location was not also repeated. Indeed, error rates indicated a significant benefit to such responses.

A location-specific SNP is consistent with several proposed theoretical explanations of negative priming. As initially suggested by Tipper et al. (1990), conflict from a distractor may provoke a direct inhibition of stimulus processing originating at that location. To the extent that such inhibition persists over time, processing of subsequent stimuli at that location would be hampered. Neill and Valdes (1992; Neill, Valdes, Terry \& Gorfein, 1992) proposed that negative priming reflects memory retrieval of the prime-processing episode. A target sharing attributes of a recent distractor might cue the retrieval of "ignore this stimulus" or "no response" or even the response made on the previous trial (Rothermund, Wentura \& De Houwer, 2005), any of which would interfere with selecting the correct response. Park and Kanwisher (1994) argued that SNP is due to a feature mismatch between the 
prime distractor and probe target. Essentially, the mismatch interferes with identification of the target as such. The latter two explanations both attribute SNP to proactive interference from characteristics of the prime trial, rather than a mechanism of direct inhibition per se.

It may be noted that responses were defined in this experiment by relative location, i.e., ordinal position. If SNP had occurred for a response that was associated with the ignored distractor, such an effect might alternatively have been attributable to repetition of relative location, rather than inhibition of the response, per se. The distinction is of course rendered moot here, as SNP depended on repetition of absolute location. This conclusion is consistent with an experiment by Tipper et al. (1990, Experiment 2). In that experiment, a prime-trial target and distractor each appeared at one of four location markers near the top of the monitor screen, while the probe-trial target and distractor each appeared at one of four other location markers near the bottom of the screen. No SNP was obtained for a probe target appearing in the same relative location as the prime-trial distractor. Tipper et al. concluded that SNP depended on absolute position, not relative position or response. Moreover, because subjects were instructed to switch eye fixation from the prime display to the probe display, the repeated relative location should correspond to the same absolute retinal location. Tipper et al. inferred that absolute environmental coordinates, not retinal coordinates, were essential to SNP. ${ }^{3}$

Our conclusion that SNP is location-specific, not responsespecific, should not be construed to imply that responses cannot be inhibited. It is quite clear that response inhibition occurs in other experimental settings, such as the "stop-signal' paradigm (Logan, 1981; Logan \& Cowan, 1984; Logan, Cowan \& Davis, 1984). The question is whether a distractor stimulus activates a response that must be inhibited, such that this inhibition persists over time and makes that same response less available to another stimulus. Insofar as the present experiment actually shows a benefit (in error rates) to a response associated with a distractor, the answer here appears to be "no."

Location-specific SNP has implications for recent discussions of differences between sensory modalities. As mentioned earlier, Wesslein et al. (2016) demonstrated a location-specific effect in touch. Because previous research

\footnotetext{
${ }^{3}$ Interpretation of the null result in Tipper et al. (1990) is rendered more problematic, because SNP was not tested at the absolute location of the prime distractor in that experiment, for comparison. Neill, Valdes, and Terry (1992) also reported a different result in a similar experiment: Whereas all eight location markers remained simultaneously in view in Tipper et al. (1990), only four markers were in view at a time in the Neill et al. experiment. In contrast to Tipper et al., we found significant SNP at the same relative location as the prime distractor. It is unclear whether the change in procedure encouraged subjects to encode locations more as relative, rather than absolute, or if subjects more successfully re-fixated the probe display, resulting in better matched retinal positions.
}

(Buckolz et al., 2004; Buckolz, Edgar et al., 2012; Guy et al., 2006) seemed to indicate that SNP in the visual modality is due to response inhibition, Wesslein et al. naturally concluded that the mechanism underlying SNP differs between touch and vision. As argued here, support for response inhibition in the visual modality is weak, and the present experiment strongly indicates a location-specific mechanism. Thus, touch and vision may in fact be more similar than previously supposed. (One must also wonder why responses per se, which are not defined by a sensory modality, should only be inhibited for stimuli in one modality.)

Interestingly, although feature-mismatch (Park \& Kanwisher, 1994) has been discredited as the sole cause of SNP in the visual modality (Milliken et al., 1994; Tipper et al., 1995), there is strong evidence for it in the auditory domain (Mayr, Hauke \& Buchner, 2009; Moller, Mayr \& Buchner, 2013). Specifically, if the designated probe-target identity is switched to match the prime-distractor identity, positive priming, rather than negative, occurs. (This is the pattern originally reported for visual displays by Park \& Kanwisher, 1994, but disputed by Milliken et al., 1994, and Tipper et al., 1995.) In contrast, tactile SNP occurs regardless of whether the probe target matches the prime distractor (Frings, Mast \& Spence, 2014; Wesslein et al., 2016).

Recent reviews of negative priming (Frings et al., 2015; May et al., 1995; Neill, 2007) have concluded that neither inhibition nor memory retrieval alone provide a sufficient account of the range of findings. Thus, it appears that more than one mechanism can underlie the effect. Although this conclusion derives largely from experiments on identity negative priming, it would be unsurprising if the same holds true for spatial negative priming. Although the parameters of the present experiment are typical of most studies of SNP (cf. the seminal experiments by Tipper and colleagues), we cannot rule out the possibility that response inhibition plays a role in some variations of the paradigm.

Of some relevance to this question is an ongoing debate over whether SNP is the same phenomenon as "inhibition of return" (IOR). In the typical demonstration of IOR, an uninformative cue attracts attention to a possible target location, and attention is then directed back to fixation (Posner \& Cohen, 1980, 1984). If the cue-target delay is more than approximately $300 \mathrm{~ms}$, RT is typically slower to a target at the cued location than to a target at an uncued location. This effect is commonly interpreted as inhibition of returning attention to a location, once attention has been withdrawn from that location. Christie and Klein (2001) argued that SNP is "really" IOR (see also Milliken et al., 2000). That is, on some proportion of prime trials, attention is accidentally directed to the distractor location, and then withdrawn in order to attend to the target location. Consequently, IOR occurs for a probe target appearing at that location. (This begs the 
question of whether IOR is "really" SNP, as suggested by Neill \& Mathis, 1998.)

In most experiments on IOR, the same responses (often detection or discrimination) are usually required for stimuli at both the cued and uncued locations. Therefore, the effect cannot be attributed to inhibition of a specific response, and so IOR appears to be truly location-specific. By extension, if SNP is "really" IOR, then SNP must be location-specific, not response-specific. Buckolz, Fitzgeorge et al. (2012) have argued that IOR and SNP are distinctly different phenomena but that IOR also may occur in some SNP experiments. In particular, they assert that IOR operates only in peripheral vision, and so only an empirical SNP in entirely foveal displays is "pure" (and, they assume, due to response inhibition). In contrast, in wider displays there is opportunity for IOR at the location of an ignored distractor. Given that the displays in the present experiment were relatively wide, it could be argued that our location-specific effect is due to IOR and is not truly SNP.

We question this line of argument on several grounds. First, in their literature review, Buckolz, Fitzgeorge et al. observed that across studies, attended-repetition trials (i.e., probe target appearing in prime target location) usually yield facilitation when displays are narrow (foveal or parafoveal), but cost when displays are wide (peripheral). They suggest that an attended-repetition benefit is a marker of conditions to produce SNP. We obtained robust facilitation in our wide-wide and narrow-narrow displays. We acknowledge that some identity priming could have compensated for IOR (Christie \& Klein, 2001; Milliken et al., 2000), but the pattern of results is nonetheless consistent with the studies that Buckolz, Fitzgeorge et al. characterize as demonstrating SNP rather than IOR.

Second, although IOR may not occur in foveal vision, there is no reason to believe that SNP should occur only in the fovea. If that were true, then most studies of SNP have not been studying "true" SNP. We would expect that, if SNP is due to response inhibition, subjects would inhibit responses to peripheral distractors as well. Yet, no response-specific SNP occurred at all in this experiment, and error rates actually suggested the opposite effect, i.e., that distractor-activated responses persist in availability. ${ }^{4}$

Third, although the span of our wide displays would be "peripheral" and our narrow displays "parafoveal," the L2 and L3 locations of the narrow displays are in the range of "central" vision used by Buckolz, Fitzgeorge et al. If pure SNP occurs only centrally, and that is due to response

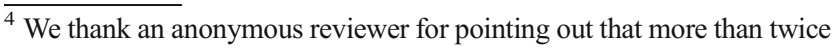
as many trials entered into the analysis of response effects than the analysis of location effects (see the Design section.) As such, subject means for the response effects should have greater reliability than the means for location effects, and thereby afford greater power a priori for detecting such effects.
}

inhibition, then half of the narrow prime trials at least should yield response-specific effect. We did find smaller (but still significant) location-specific SNP in the narrow-wide sequences than in the wide-narrow sequences. (We attributed this to a floor effect on RT.) However, there was no concomitant increase of a response-specific effect in those sequences.

Fourth, and most important, SNP and IOR are empirical phenomena, defined by a pattern of results in a particular experimental paradigm. There is no strong agreement on what mechanisms underlie either of these phenomena. Neill and Mathis (1998) in fact opined that IOR should be regarded as a form of negative priming, insofar as the latter is a theoretically neutral term: It is operationally defined by slower or less accurate responses to a stimulus sharing some attribute of a previous stimulus. (In contrast, the phrase "inhibition of return" presupposes a mechanism underlying the observed pattern.) The literature strongly points to multiple causes of identity negative priming (Frings et al., 2015; May et al., 1995; Neill, 2007), and the same may well be true of both SNP and IOR. (Regarding the latter, see review by Berlucchi, 2006.) SNP and IOR may sometimes reflect the same causal mechanism, sometimes not. It is too dogmatic to label a pattern of results attributed to one mechanism as "really" SNP or IOR and to dismiss a very similar pattern of results attributed to a different mechanism as not the genuine thing. A more sensible strategy is to try to determine the circumstances in which a pattern of results occurs, regardless of what we call it.

Acknowledgments Portions of this research were presented at the $14^{\text {th }}$ annual meeting of the Vision Sciences Society, May, 2014, St. Pete Beach, FL. The authors thank Daniela Azaraev, Sabrina Cox, Roy Harding, Joslin Kennedy, Camila Osses, and Mehwish Shahid for assistance with data collection.

\section{References}

Berlucchi, G. (2006). Inhibition of return: a phenomenon in search of a mechanism and a new name. Cognitive Neuropsychology, 23, 10651074.

Buckolz, E., Edgar, C., Kajaste, B., Lok, M., \& Khan, M. (2012). Inhibited prime-trial distractor responses solely produce the visual spatial negative priming effect. Attention, Perception, \& Psychophysics, 74, 1632-1643.

Buckolz, E., Fitzgeorge, L., \& Knowles, S. (2012). Spatial negative priming, but not inhibition of return, with central (foveal) displays. Psychology, 3, 666-674.

Buckolz, E., Goldfarb, A., \& Khan, M. (2004). The use of a distractorassigned response slows later responding in a location negativepriming task. Perception \& Psychophysics, 66, 837-845.

Christie, J., \& Klein, R. M. (2001). Negative priming for spatial locations? Canadian Journal of Experimental Psychology, 55, 24-38.

Frings, C., Mast, F., \& Spence, C. (2014). Tactile spatial negative priming without feature mismatch. Attention, Perception, \& Psychophysics, 76, 2305-2314. 
Frings, C., Schneider, K. K., \& Fox, E. (2015). The negative priming paradigm: An update and implications for selective attention. Psychonomic Bulletin \& Review, 22, 1577-1597.

Guy, S., Buckolz, E., \& Khan, M. (2006). The locus of location repetition latency effects. Canadian Journal of Experimental Psychology, 60, 307-318.

Logan, G. D. (1981). Attention, automaticity, and the ability to stop a speeded choice response. In J. Long \& A. D. Baddeley (Eds.), Attention and performance IX (pp. 205-222). Hillsdale, NJ: Erlbaum.

Logan, G. D., \& Cowan, W. B. (1984). On the ability to inhibit thought and action: A theory of an act of control. Psychological Review, 91, 295-397.

Logan, G. D., Cowan, W. B., \& Davis, K. A. (1984). On the ability to inhibit simple and choice reaction time responses: A model and a method. Journal of Experimental Psychology: Human Perception and Performance, 10, 276-291.

Lowe, D. G. (1979). Strategies, context, and the mechanism of response inhibition. Memory \& Cognition, 7, 382-389.

May, C. P., Kane, M. J., \& Hasher, L. (1995). Determinants of negative priming. Psychological Bulletin, 118, 35-54.

Mayr, S., Hauke, R., \& Buchner, A. (2009). Auditory location negative priming: A case of feature mismatch. Psychonomic Bulletin \& Review, 16, 845-849.

Milliken, B., Joordens, S., Merikle, P., \& Seiffert, A. (1998). Selective attention: A re-evaluation of the implications of negative priming. Psychological Review, 105, 203-229.

Milliken, B., Tipper, S. P., Houghton, G., \& Lupianez, J. (2000). Attending, ignoring and repetition: On the relation between negative priming and inhibition of return. Perception \& Psychophysics, 62, 1280-1296.

Milliken, B., Tipper, S. P., \& Weaver, B. (1994). Negative priming in a spatial localization task: Feature mismatching and distractor inhibition. Journal of Experimental Psychology: Human Perception and Performance, 20, 624-646.

Moller, M., Mayr, S., \& Buchner, A. (2013). Target localization among concurrent sound sources: No evidence for the inhibition of previous distractor responses. Attention, Perception, \& Psychophysics, 75, $132-144$.

Moore, C. M. (1994). Negative priming depends on probe-trial conflict: Where has all the inhibition gone? Perception \& Psychophysics, 56, 133-147.

Neely, J. H. (1977). Semantic priming and retrieval from lexical memory: Roles of inhibitionless spreading activation and limited-capacity attention. Journal of Experimental Psychology: General, 106, 226254.

Neill, W. T. (1977). Inhibitory and facilitatory processes in selective attention. Journal of Experimental Psychology: Human Perception and Performance, 3, 444-450.

Neill, W. T. (2007). Mechanisms of transfer-inappropriate processing. In D. S. Gorfein \& C. M. MacLeod (Eds.), Inhibition in cognition. Washington, DC: American Psychological Association.

Neill, W. T., \& Mathis, K. M. (1998). Transfer-inappropriate processing: Negative priming and related phenomena. In D. Medin (Ed.), The psychology of learning and motivation: Advances in research and theory (Vol. 38, pp. 1-44). San Diego, CA: Academic Press.

Neill, W. T., Terry, K. M., \& Valdes, L. A. (1994). Negative priming without probe selection. Psychonomic Bulletin \& Review, 1, 119121.

Neill, W. T., \& Valdes, L. A. (1992). Persistence of negative priming: Steady-state or decay? Journal of Experimental Psychology: Learning, Memory, and Cognition, 18, 565-576.
Neill, W. T., \& Valdes, L. A. (1996). Facilitatory and inhibitory aspects of attention. In A. F. Kramer, M. G. H. Coles, \& G. D. Logan (Eds.), Converging operations in the study of visual selective attention (pp. 77-106). Washington, DC: American Psychological Association.

Neill, W. T., Valdes, L. A., Terry, K. M., \& Gorfein, D. S. (1992). The persistence of negative priming: II. Evidence for episodic trace retrieval. Journal of Experimental Psychology: Learning, Memory, and Cognition, 18, 993-1000.

Neill, W. T., Valdes, L. A., \& Terry, K. M. (1995). Selective attention and the inhibitory control of cognition. In F. N. Dempster \& C. J. Brainerd (Eds.), Interference and inhibition in cognition (pp. 207261). San Diego: Academic Press.

Neill, W. T., Valdes. L. A., \& Terry, K. M. (1992, November). Negative priming in target localization. Paper presented at the meeting of the Psychonomic Society, St. Louis, MO.

Park, J., \& Kanwisher, N. (1994). Negative priming for spatial locations: Identity mismatching, not distractor inhibition. Journal of Experimental Psychology: Human Perception and Performance, 20, 613-623.

Posner, M. I., \& Cohen, Y. (1980, November). Consequences of visual orienting. Paper presented at the meeting of the Psychonomic Society, St. Louis, MO.

Posner, M. I., \& Cohen, Y. (1984). Components of visual orienting. In H. Bouma \& D. G. Bouwhuis (Eds.), Attention and performance X: Control of language processes (pp. 531-556). Hillsdale, NJ: Erlbaum.

Posner, M. I., \& Snyder, C. R. R. (1975). Facilitation and inhibition in the processing of signals. In P. M. A. Rabbitt \& S. Dornic (Eds.), Attention and performance $V$ (pp. 669-682). New York: Academic Press.

Rothermund, K., Wentura, D., \& De Houwer, J. (2005). Retrieval of incidental stimulus-response associations as a source of negative priming. Journal of Experimental Psychology: Learning, Memory, and Cognition, 31, 482-495.

Stroop, J. R. (1935). Studies of interference in serial verbal reactions. Journal of Experimental Psychology, 18, 643-662.

Tipper, S. P. (1985). The negative priming effect: Inhibitory priming by ignored objects. Quarterly Journal of Experimental Psychology, 37, 571-590.

Tipper, S. P., Brehaut, J., \& Driver, J. (1990). Selection of moving and and static objects for the control of spatially directed action. Journal of Experimental Psychology: Human Perception and Performance, 16, 492-504.

Tipper, S. P., \& Cranston, M. (1985). Selective attention and priming: Inhibitory and facilitatory effects of ignored primes. Quarterly Journal of Experimental Psychology, 37, 591-611.

Tipper, S. P., Lortie, C., \& Baylis, G. (1992). Selective reaching: Evidence for action-centered attention. Journal of Experimental Psychology: Human Perception and Performance, 18, 891-905.

Tipper, S. P., Weaver, B., Cameron, S., Brehaut, J. C., \& Bastedo, J. (1991). Inhibitory mechanisms of attention in identification and localization tasks: Time course and disruption. Journal of Experimental Psychology: Learning, Memory, and Cognition, 17, 681-692.

Tipper, S. P., Weaver, B., \& Milliken, B. (1995). Spatial negative priming without mismatching: Comment on Park and Kanwisher (1994). Journal of Experimental Psychology: Human Perception and Performance, 21, 1220-1229.

Wesslein, A.-K., Spence, C., Mast, F., \& Frings, C. (2016). Spatial negative priming: In touch, it's all about location. Attention, Perception, \& Psychophysics, 78, 464-473. 